\title{
Current reversals in a rocking ratchet: The frequency domain
}

\author{
A. Wickenbrock, ${ }^{1}$ D. Cubero, ${ }^{2}$ N. A. Abdul Wahab, ${ }^{1}$ P. Phoonthong, ${ }^{1}$ and F. Renzoni ${ }^{1}$ \\ ${ }^{1}$ Department of Physics and Astronomy, University College London, Gower Street, London WC1E 6BT, United Kingdom \\ ${ }^{2}$ Departamento de Física Aplicada I, EUP, Universidad de Sevilla, Calle Virgen de África 7, E-41011 Sevilla, Spain, and Física Teórica, \\ Universidad de Sevilla, Apartado de Correos 1065, Sevilla E-41080, Spain
}

(Received 10 May 2011; published 18 August 2011)

\begin{abstract}
Motivated by recent work [D. Cubero et al., Phys. Rev. E 82, 041116 (2010)], we examine the mechanisms which determine current reversals in rocking ratchets as observed when varying the frequency of the drive. We found that a class of these current reversals in the frequency domain is precisely determined by dissipation-induced symmetry breaking. Our experimental and theoretical work thus extends and generalizes the previously identified relationship between dynamical and symmetry-breaking mechanisms in the generation of current reversals.
\end{abstract}

DOI: 10.1103/PhysRevE.84.021127

PACS number(s): 05.40.-a, 05.45.-a, 05.60.-k

\section{INTRODUCTION}

In out-of-equilibrium systems, directed transport can be obtained without the application of a net bias force. Such a counterintuitive phenomenon is usually termed the ratchet effect [1-8]. The occurrence of a directed current in such systems can be precisely related to the breaking of the relevant spatiotemporal symmetries [9-11].

An intriguing feature of ratchets is current reversals, where the sign of the generated current changes following a variation in a system parameter $[4,12,13]$. As a specific example, consider the case of a rocking ratchet, consisting of Brownian particles in an asymmetric sawtooth potential. A sinusoidal rocking force drives the system out of equilibrium, thus allowing the generation of directed transport. In such a system, current reversals can be observed by varying the noise strength at fixed amplitude of the rocking force, as well as by varying the force amplitude while keeping constant the noise strength [4]. The key feature of current reversals is that the sign of the current can be reversed by varying a system parameter, although the considered parameter does not change the symmetry of the Hamiltonian. At first glance, this suggests that current reversals are a dynamical phenomenon, not traceable back to a symmetry-breaking mechanism.

Previous theoretical work aimed to identify the mechanisms underlying current reversals in rocking ratchets. For an underdamped deterministic ratchet, Mateos [13] argued that current reversals induced by a variation of the driving strength correspond to a bifurcation from a chaotic to a periodic regime. However, the proposed mechanisms turned out not to be general, as in the very same system current reversals can be observed also in the absence of such bifurcations, and moreover not all chaos-to-order transitions necessarily lead to current reversals [14,15].

A different approach toward understanding current reversals induced by a variation of the rocking force amplitude was recently introduced for a biharmonically driven, spatially symmetric rocking ratchet [16]. It was shown that a class of current reversals is precisely determined by symmetry breaking. In this way, a link was established between dynamical and symmetry-breaking mechanisms. The issue still open is to what extent this link can be generalized, either to different systems or to current reversals of different type in the same setup.
In the present work, we generalize the link between dynamical and symmetry-breaking mechanisms introduced in the aforementioned work. We consider the same ratchet system and examine the current reversals induced by a variation of the frequency of the rocking force. We notice that a variation of the rocking-force frequency cannot be mapped onto a variation of the force amplitude, that is, the current reversals considered here are not a priori equivalent to the ones observed by varying the force strength. Our experimental and theoretical work shows that also in this case there is a class of current reversals determined by symmetry breaking, thus generalizing the argument put forward in Ref. [16].

This work is organized as follows. In Sec. II, we introduce the ratchet setup used in this work and recall the elements of the symmetry analysis that will be needed to establish the link between current reversals in the frequency domain and dissipation-induced symmetry breaking. Section III presents experimental results obtained with cold atoms in a driven optical lattice in the regime of weak damping. Section IV analyzes theoretically the relationship between dynamical and symmetry-breaking mechanisms for the current reversals induced by a variation in the driving frequency. Conclusions are drawn in Sec. V.

\section{SETUP AND SYMMETRIES}

\section{A. Ratchet setup}

In this work, we consider a ratchet setup consisting of Brownian particles in a spatially symmetric periodic potential driven by a time-asymmetric force. To capture the main features of the dynamics, in the theoretical analysis we consider the simple case of a linear friction, as usually considered in the ratchet literature. This also allows us to explore different regimes of damping and noise. The relevant Langevin equation is in this case

$$
m \ddot{x}=-\alpha \dot{x}-U^{\prime}(x)+F(t)+\xi(t),
$$

where $U(x)=U_{0} \cos (2 k x) / 2$ is a periodic potential, $\alpha$ is the friction coefficient, $\xi(t)$ is a Gaussian white noise $\langle\xi(t)\rangle=$ $0,\left\langle\xi(t) \xi\left(t^{\prime}\right)\right\rangle=2 D \delta\left(t-t^{\prime}\right)$, and $F(t)$ is a biharmonic drive described by

$$
F(t)=F_{0}\left[A_{1} \cos (\omega t)+A_{2} \cos (2 \omega t+\phi)\right] .
$$


The appearance of a ratchet effect, that is, the generation of directed motion, in such a setup is very well established [17-22].

\section{B. Symmetry analysis}

We now recall the essential elements of the symmetry analysis, initially developed to explain the generation of a current [9-11], that will allow us to introduce the basic concepts needed to establish the link between current reversals in the frequency domain and dissipation-induced symmetry breaking.

In general, the symmetry analysis [9-11] is used to identify the symmetry of the system which prevent directed motion. For the specific case of a spatially symmetric potential of interest here, there are two of those symmetries: the shift symmetry, which corresponds to invariance under the transformation $(x, p, t) \rightarrow(-x,-p, t+T / 2)$, with $T$ the period of the drive, and the time-reversal symmetry, which requires invariance under the transformation $(x, p, t) \rightarrow(x,-p,-t)$. A biharmonic drive of the form of Eq. (2) breaks the shift symmetry for any value of the relative phase $\phi$. For the time-reversal symmetry, it is necessary to distinguish different cases, corresponding to different levels of dissipation. For no dissipation (Hamiltonian case), the system is symmetric under time reversal for $\phi=n \pi$ with $n$ integer, and therefore for these values directed motion cannot be produced. It can be shown $[9,23]$ that the dependence of the average velocity on the phase $\phi$ is, in leading order, $v=A \sin \phi$. Consider now the case of nonzero dissipation. For nonzero dissipation, the time-reversal symmetry is broken by dissipation also for $\phi=n \pi$ with $n$ integer. Thus, a current can be generated also for these values of the phase $\phi$. For weak dissipation, the dependence of the average velocity on the phase $\phi$ is, in leading order, $v=A \sin \left(\phi-\phi_{0}\right)$, where $\phi_{0}$ is a dissipation-induced symmetry-breaking phase lag which vanishes in the Hamiltonian limit [10,23,24]. Finally, in the overdamped regime, the system is invariant under the so-called supersymmetry [11] $(x, p, t) \rightarrow(x+\lambda / 2,-p,-t)$, with $\lambda$ the spatial period of the potential, for $\phi=\pi / 2+n \pi$, with $n$ integer. For these values of the phase $\phi$, direction motion is not allowed.

\section{EXPERIMENTAL RESULTS}

The experimental setup and procedure are substantially the same as the ones used in our previous work of Ref. [16], and we recall here only the essential elements. Up to $10^{8}{ }^{87} \mathrm{Rb}$ atoms are trapped and cooled down to $\sim 50 \mu K$ in a magneto-optical trap. The atoms are then loaded in a one-dimensional (1D) lin $\perp$ lin dissipative optical lattice, in which the atom-light interaction determines both a periodic potential for the atoms and the dissipation mechanism, which leads to a friction force and to fluctuations in the atomic dynamics. A driving of the form of Eq. (2) is applied by phase modulating one of the lattice beams [25]. For all the measurements presented in this work, the ratio between harmonics is kept fixed: $A_{1}=2, A_{2}=1$. The motion of the atoms in the driven lattice is studied by imaging the atomic cloud with a charge-coupled device (CCD) camera. The velocity of the center of mass of the atomic cloud is then derived using these images.

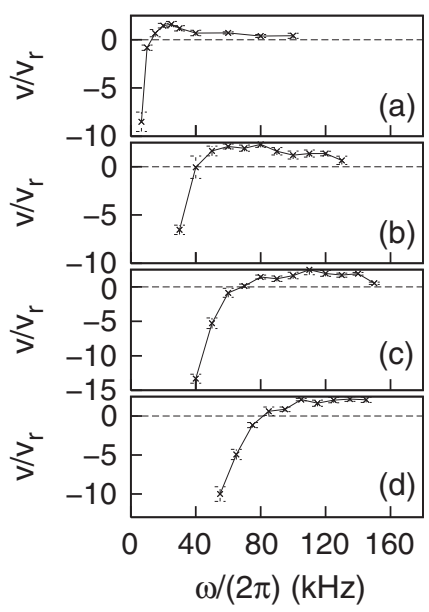

FIG. 1. Experimental results for 1D rocking ratchet for cold atoms. The average atomic velocity, rescaled by the recoil velocity $v_{r}\left(v_{r}=5.88 \mathrm{~mm} / \mathrm{s}\right.$ for $\left.{ }^{87} \mathrm{Rb}\right)$, is reported as a function of the frequency of the drive, for a fixed value of the relative phase between harmonics of the ac drive. The different data sets correspond to different amplitudes of the driving force. The parameters of the optical lattice are detuning from resonance $\Delta=-9 \Gamma$ and intensity per lattice beam $I_{L}=(43.5 \pm 0.3) \mathrm{mW} / \mathrm{cm}^{2}$. The driving is a biharmonic force of the form of Eq. (2), with the relative phase kept fixed at $\phi=\pi / 2$ for the presented sets of measurements. The amplitude of the force is $F_{0}=-m \lambda g_{0}$ where $m$ is the atomic mass, $\lambda$ is the laser field wavelength, and the values for $g_{0}$ (in $\mathrm{kHz}^{2}$ ) are, for the different set of data reported in the figures, (a) $g_{0}=3.2 \times 10^{3}$, (b) $g_{0}=12.8 \times 10^{3}$, (c) $g_{0}=19.2 \times 10^{3}$, and (d) $g_{0}=25.6 \times 10^{3}$. The lines are guides for the eye.

We first consider the standard configuration for the detection of current reversals: For a fixed Hamiltonian, with broken symmetry, the current is studied as a function of a parameter whose variation does not change the symmetry of the Hamiltonian. In the present case, we fix the relative phase between driving harmonics $\phi=\pi / 2$, so to break the time-reversal symmetry, and study the current as a function of the driving frequency $\omega$. Results of our experiment are shown in Fig. 1. The familiar situation of current reversals in the frequency domain $[21,26]$ is observed: By varying the frequency of the drive it is possible to revert the direction of the atomic current through the lattice.

We now study the atomic current as a function of the phase $\phi$ for different values of the driving frequency, in a range of frequencies around the value at which the current reversal is observed. This will allow us to establish a relationship between the observed current reversal and dissipative effects.

The results of our measurements are shown in Fig. 2, for a driving force amplitude corresponding to that of Fig. 1(c). For all considered driving frequencies, the dependence of the current on the phase $\phi$ is well described by $v / v_{r}=A \sin (\phi-$ $\phi_{0}$ ), with $\phi_{0}$ a dissipation-induced symmetry-breaking phase lag. This is in agreement with previous observations [16,24]. The important, and so far unexplored, fact here is that $\phi_{0}$ varies significantly when the driving frequency is scanned across the value corresponding to the current reversal in the frequency domain. 


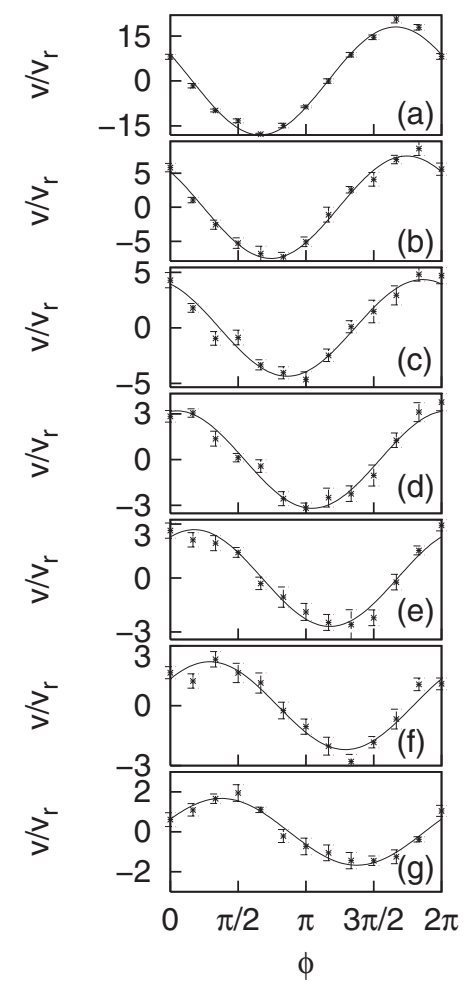

FIG. 2. Experimental results for 1D rocking ratchet for cold atoms. The driving is a biharmonic force of the form of Eq. (2). The average atomic velocity, rescaled by the recoil velocity $v_{r}$, is plotted as a function of the relative phase $\phi$ between harmonics of the ac drive, for different values of the driving force frequency. The lines are the best fit of the data with the function $v / v_{r}=A \sin \left(\phi-\phi_{0}\right)$. The lattice parameters are the same as in Fig. 1. The frequencies of the drive for the different data sets are (a) $\omega /(2 \pi)=40 \mathrm{kHz}$, (b) $\omega /(2 \pi)=50 \mathrm{kHz}$, (c) $\omega /(2 \pi)=60 \mathrm{kHz}$, (d) $\omega /(2 \pi)=70 \mathrm{kHz}$, (e) $\omega /(2 \pi)=80 \mathrm{kHz}$, (f) $\omega /(2 \pi)=100 \mathrm{kHz}$, and $(\mathrm{g}) \omega /(2 \pi)=120 \mathrm{kHz}$. The driving force amplitude, for all data sets, is $F_{0}=-m \lambda g_{0}$ with $g_{0}=19.2 \times 10^{3} \mathrm{kHz}^{2}$.

The dependence of the phase lag $\phi_{0}$ and the amplitude $A$, as obtained by fitting data as those of Fig. 2 with the function $v / v_{r}=A \sin \left(\phi-\phi_{0}\right)$, on the driving frequency is reported in Fig. 3. The four different data sets correspond to the four different driving-strength amplitudes for which the data in Fig. 1 were obtained. The phase lag $\phi_{0}$ shows a very large variation around the frequency at which the current reversal is observed in Fig. 1, with the phase lag varying from $-\pi$ to 0 around the current reversal and taking the value $-\pi / 2$ at the reversal frequency [27]. Since in the experiments reported in Fig. 1 the relative phase is fixed to $\phi=\pi / 2$, this value of the phase lag, $\phi_{0}=-\pi / 2$, guarantees that the current is suppressed at the reversal frequency, where the directed current inverts its sign. On the other hand, the amplitude of the sinelike curve, see right panels in Fig. 3, stays finite around the reversal frequency. Thus, we can conclude that the current reversal in the frequency domain is determined by the large variation, around the reversal frequency, of the dissipation-induced symmetry-breaking phase lag $\phi_{0}$. This generalizes the link between symmetry breaking and current reversals established in Ref. [16] in the case of current reversals in the amplitude domain.

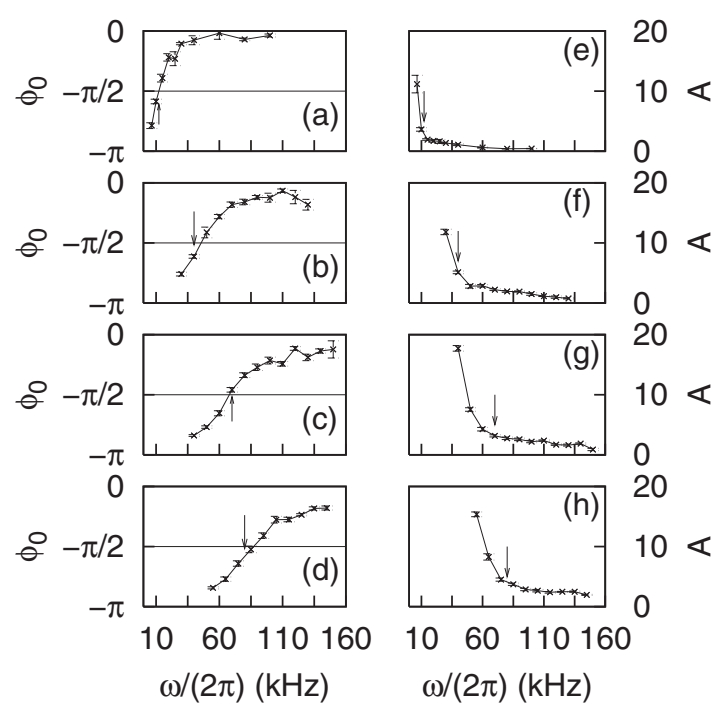

FIG. 3. (a)-(d) Dissipation-induced phase lag $\phi_{0}$, as obtained by fitting data as those in Fig. 2, with the function $v / v_{r}=A \sin \left(\phi-\phi_{0}\right)$, as a function of the frequency of the drive, for different values of the driving strength. (e)-(h) Amplitude $A$, obtained from the same fits. Different rows of plots of the figure correspond to different driving strengths, with the amplitudes of the drive the same as in Fig. 1; e.g., the data in (a) and (e) correspond to a driving amplitude $g_{0}=3.2 \times 10^{3} \mathrm{kHz}^{2}$ and so on. The lattice parameters are the same as in Fig. 1. The lines are guides for the eye. The arrows indicate the frequency at which the current reversal shown in Fig. 1 occurs.

\section{NUMERICAL ANALYSIS}

The presented experimental results show that the current reversals in the frequency domain are determined by a sharp variation of the dissipative phase lag $\phi_{0}$ when the driving frequency is varied around the value corresponding to the current reversal.

The conditions of our experiment correspond to the weakly damped regime, and hence the appearance of a nonzero dissipation-induced phase lag $\phi_{0}$. The validity of the established link between current reversals and dissipationinduced symmetry breaking can be tested by considering the Hamiltonian and the overdamped limit. In both cases, the phase lag $\phi_{0}$ is fixed by the symmetries of the system valid in the respective limits: $\phi_{0}=0$ for the Hamiltonian limit and $\phi_{0}=-\pi / 2$ [28] for the overdamped case, as discussed in Sec IIB. Thus $\phi_{0}$ cannot vary with the driving frequency, and the current reversals observed in the case of moderate dissipation should disappear.

Our experimental setup is not suitable for exploring the two extreme cases of no dissipation and very large dissipation. Thus, in order to show that these current reversals vanish when the phase lag $\phi_{0}$ is fixed by the symmetries in the Hamiltonian and overdamped regimes, we resort to numerical simulations of Eq. (1).

Let us examine first the weakly damped regime with this model. The middle panel of Fig. 4 shows the average particles' velocity as a function of the driving phase for different values of the driving frequency. A current reversal can be clearly observed at about $\phi=\pi / 2$ (or $\phi=3 \pi / 2$ ) as the driving frequency is increased, being a consequence of a variation 


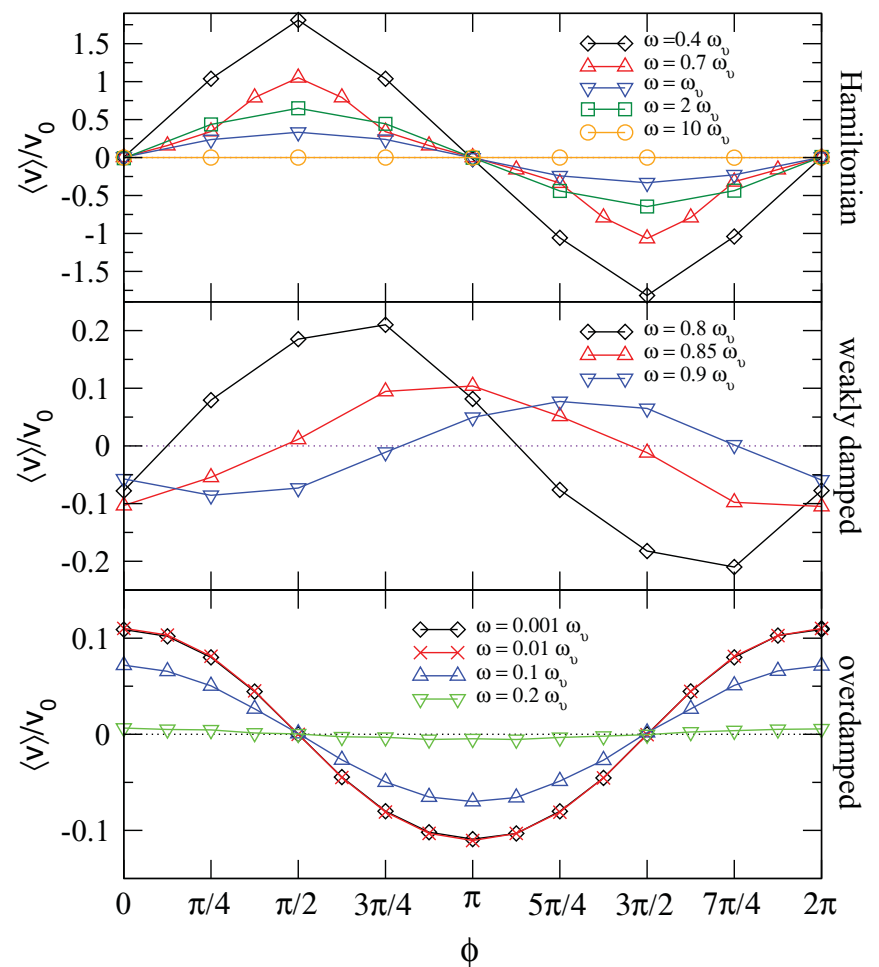

FIG. 4. (Color online) Average atomic velocity as a function of the relative phase $\phi$ between harmonics of the ac drive for several values of the driving frequency $\omega$, with $\omega_{v}=k\left(2 U_{0} / m\right)^{1 / 2}$ and $A_{1}=A_{2}=1$. Top panel: simulation data in the Hamiltonian regime $(\alpha=D=0)$ for $F_{0}=0.2 U_{0} k$. Middle panel: simulation data in the weakly damped regime. The driving amplitude, the friction, and the noise strength values are fixed to $F_{0}=0.2 U_{0} k, \alpha=0.15 \alpha_{0}$, and $D=1.944 D_{0}$, respectively, where $\alpha_{0}=m k v_{0}, D_{0}=\alpha_{0}^{2} v_{0} / k$, and $v_{0}=\left(U_{0} / m\right)^{1 / 2} / 10$. The values about $\phi=\pi / 2$ (or $\phi=3 \pi / 2$ ) show a current reversal as the driving frequency is increased. Bottom panel: simulation data in the overdamped regime for $F_{0}=U_{0} k, \alpha=100 \alpha_{0}$, and $D=1.944 \times 10^{3} D_{0}$. Lines are guides for the eye.

of the phase lag $\phi_{0}$ with the driving frequency. This is the regime explored in our experiment.
On the other hand, the phase lag $\phi_{0}$ is fixed to 0 in the Hamiltonian regime due to the time-reversal symmetry. Accordingly, the top panel of Fig. 4 shows that no current reversals are observed when the driving frequency is varied. In the limit of strong dissipation, the appearance of the supersymmetry fixes the phase lag to $\phi_{0}=-\pi / 2$, as shown in the bottom panel of Fig. 4. This fact prevents the occurrence of the current reversals observed in the presence of moderate dissipation.

The numerical simulations thus confirm the validity of the established link between current reversals in the frequency domain and dissipation-induced symmetry breaking.

\section{CONCLUSIONS}

In conclusion, we examined the mechanisms which determine current reversals in rocking ratchets as observed by varying the frequency of the drive. We considered the specific case of a biharmonically driven, spatially symmetric ratchet. We found that a class of these current reversals in the frequency domain is precisely determined by dissipationinduced symmetry breaking. Correspondingly, these reversals are observed only for moderate dissipation and disappear in the Hamiltonian and overdamped limit.

Our experimental and theoretical work thus extends and generalizes the previously identified relationship [16] between dynamical and symmetry-breaking mechanisms in the generation of current reversals.

In the future, it would be interesting to re-examine the issue of the relationship between microscopic dynamics and current reversals in the system studied by Mateos [13]. Whether the current reversals discussed there can be associated to some distinctive features of the microscopic dynamics is still an open question.

\section{ACKNOWLEDGMENTS}

This research was supported by the Leverhulme Trust. One of us (D.C.) also thanks the Ministerio de Ciencia e Innovación of Spain for financial support (Grant No. FIS2008-02873).
[1] A. Ajdari and J. Prost, C. R. Acad. Sci. Paris 315, 1635 (1992).

[2] M. O. Magnasco, Phys. Rev. Lett. 71, 1477 (1993).

[3] A. Adjari, D. Mukamel, L. Peliti, and J. Prost, J. Phys. I (France) 4, 1551 (1994).

[4] R. Bartussek, P. Hänggi, and J. G. Kissner, Europhys. Lett. 28, 459 (1994).

[5] C. R. Doering, W. Horsthemke, and J. Riordan, Phys. Rev. Lett. 72, 2984 (1994).

[6] D. Cubero, J. Casado-Pascual, A. Alvarez, M. Morillo, and P. Hänggi, Acta Phys. Pol. B 37, 1467 (2006)

[7] P. Reimann, Phys. Rep. 361, 57 (2002).

[8] P. Hänggi and F. Marchesoni, Rev. Mod. Phys. 81, 387 (2009).

[9] S. Flach, O. Yevtushenko, and Y. Zolotaryuk, Phys. Rev. Lett. 84, 2358 (2000).
[10] O. Yevtushenko, S. Flach, Y.Zolotaryuk, and A. A. Ovchinnikov, Europhys. Lett. 54, 141 (2001).

[11] P. Reimann, Phys. Rev. Lett. 86, 4992 (2001).

[12] P. Jung, J. G. Kissner, and P. Hänggi, Phys. Rev. Lett. 76, 3426 (1996).

[13] J. L. Mateos, Phys. Rev. Lett. 84, 258 (2000).

[14] M. Barbi and M. Salerno, Phys. Rev. E 62, 1988 (2000).

[15] A. Kenfack, S. M. Sweetnam, and A. K. Pattanayak, Phys. Rev. E 75, 056215 (2007).

[16] D. Cubero, V. Lebedev, and F. Renzoni, Phys. Rev. E 82, 041116 (2010).

[17] F. Marchesoni, Phys. Lett. A 119, 221 (1986).

[18] D. R. Chialvo and M. M. Millonas, Phys. Lett. A 209, 26 (1996).

[19] M. I. Dykman, H. Rabitz, V. N. Smelyanskiy, and B. E. Vugmeister, Phys. Rev. Lett. 79, 1178 (1997). 
[20] I. Goychuk and P. Hänggi, Europhys. Lett. 43, 503 (1998).

[21] D. G. Luchinsky, M. J. Greenall, and P. V. E. McClintock, Phys. Lett. A 273, 316 (2000).

[22] L. Machura, M. Kostur, and J. Luczka, Chem. Phys. 375, 445 (2010).

[23] N. R. Quintero, J. A. Cuesta, and R. Alvarez-Nodarse, Phys. Rev. E 81, 030102(R) (2010).

[24] R. Gommers, S. Bergamini, and F. Renzoni, Phys. Rev. Lett. 95, 073003 (2005).

[25] F. Renzoni, Adv. At. Mol. Opt. Phys. 57, 1 (2009).
[26] R. Gommers, P. Douglas, S. Bergamini, M. Goonasekera, P. H. Jones, and F. Renzoni, Phys. Rev. Lett. 94, 143001 (2005).

[27] Not all the plots in the figure show exactly $\phi_{0}=\pi / 2$. This is due to a measurement error, as $\phi_{0}$ is determined by fitting the entire sinelike curve obtained by varying $\phi$ while the reversal frequency is determined by measuring only the current for $\phi=\pi / 2$.

[28] Note that the fact that the phase lag is $\phi_{0}=-\pi / 2$ and not $\phi_{0}=\pi / 2$ (assuming a positive $A$ ) cannot be determined a priori by the symmetry analysis, and here is reported based on the a posteriori observation of the numerical results. 